\title{
Effects of cluster headache preventatives on mouse hypothalamic transcriptional homeostasis
}

Cephalalgia

0(0) I-6

(C) International Headache Society 2022

Article reuse guidelines:

sagepub.com/journals-permissions

DOI: $10.1177 / 03331024221075613$

journals.sagepub.com/home/cep

(SAGE

\author{
Alessandra Pistolesi*, Daniela Buonvicino*, Mirko Muzzi, \\ Matteo Urru and Alberto Chiarugi
}

\begin{abstract}
Objective: To investigate how cluster headache preventatives verapamil, lithium and prednisone affect expression of hypothalamic genes involved in chronobiology.

Methods: C57BI/6 mice were exposed to daily, oral treatment with verapamil, lithium, prednisone or amitriptyline (as negative control), and transcripts of multiple genes quantified in the anterior, lateral and posterior hypothalamus.

Results: Verapamil, lithium or prednisone did not affect expression of clock genes of the anterior hypothalamus (Clock, Bmall, Cryl/2 and Perl/2). Prednisone altered expression of hypothalamic neuropeptides melanin-concentrating hormone and histidine decarboxylase within the lateral and posterior hypothalamus, respectively. The three preventatives did not affect expression of the neurohypophyseal hormones oxytocin and arginine-vasopressin in the posterior hypothalamus. Conversely, amitriptyline reduced mRNA levels of Clock, oxytocin and arginine-vasopressin.

Conclusion: Data suggest that cluster headache preventatives act upstream or downstream from the hypothalamus. Our findings provide new insights on hypothalamic homeostasis during cluster headache prophylaxis, as well as neurochemistry underlying cluster headache treatment.
\end{abstract}

\section{Keywords}

Cluster headache, verapamil, lithium, prednisone, hypothalamus, transcriptional homeostasis

Date received: 10 September 202 I; revised: 29 November 202I; accepted: 14 December 2021

\section{Introduction}

Cluster headache $(\mathrm{CH})$ has a prevalence of 1 in 1000 and a male/female ratio of 2.5-3.5. Rhythmicity represents one of the most enigmatic feature of $\mathrm{CH}$, and a great deal of effort has been directed at deciphering the underlying molecular mechanisms. Nevertheless, the neurobiology of the circadian/circannual pattern of $\mathrm{CH}$ and its relationship with attack pathogenesis still waits to be deciphered (1). Because of the key role of the hypothalamus in human chronobiology, as well as in the pathogenesis of primary headaches in general, it has been repeatedly proposed that this brain region is causally involved in $\mathrm{CH}$ rhythmicity and pain (2). Accordingly, several fMRI studies report hypothalamic activation during $\mathrm{CH}$ attacks (3). Further confirming a pivotal role of the hypothalamus in the etiopathogenesis of $\mathrm{CH}$, deep brain stimulation with electrodes implanted in the posterior hypothalamus reduces both severity and frequency of attacks in $\mathrm{CH}$ patients refractory to pharmacological therapy (4).

Verapamil, lithium, and corticosteroids represent the mainstay of $\mathrm{CH}$ prophylaxis, but how they function as preventatives is unknown. In this regard, it is worth noting that from a pharmacodynamic perspective, $\mathrm{CH}$ preventatives do not show any apparent, mutual mechanism of action. Specifically, verapamil is a calcium

Department of Health Sciences, Section of Clinical Pharmacology and Oncology, University of Florence, Florence, Italy

*These authors contributed equally to this work.

\section{Corresponding author:}

Alessandra Pistolesi, Department of Health Sciences, Section of Clinical Pharmacology and Oncology, University of Florence, Viale Pieraccini 6, 50139 Florence, Italy.

Email: alessandra.pistolesi@unifi.it 
channel blocker approved as an antiarrhythmic, lithium is an inositol phosphatase/glycogen synthase kinase inhibitor used as a mood stabiliser, and corticosteroids are transcription-regulating, immunomodulatory drugs. Notwithstanding the lack of similar pharmacodynamics, to our knowledge the question as to whether these drugs share a similar impact on hypothalamic functions is still unanswered. We also reasoned that a deeper understanding of the pharmacological mechanisms underlying $\mathrm{CH}$ prophylaxis can help developing innovative therapeutics, for which patients are in greet need.

Hence, in an attempt to gather information on $\mathrm{CH}$ pathogenesis and molecular mechanisms underpinning prophylaxis, in the present study we investigated whether oral treatment with verapamil, lithium carbonate or prednisone alter expression profiles of genes involved in hypothalamic functioning, circadian clock and sleep regulation in mice.

\section{Materials and methods}

\section{Animals}

All animal care and experimental procedures were performed according to the European Community guidelines for animal care (European Communities Council Directive 2010/63/EU) and were approved by the Committee for Animal Care and Experimental Use of the University of Florence. Male C57Bl/6 mice 20-25 g were purchased from Charles River (Milan, Italy) and housed in conventional units (six per cage) with free access to food (Harlan Global Diet 2018, Harlan Laboratories, Udine, Italy) and water. Mice were maintained on a $12 \mathrm{~h} \mathrm{light/dark} \mathrm{cycle} \mathrm{at} 21^{\circ} \mathrm{C}$ room temperature for 15 days before drug exposure. As for the power analysis, the experimental hypothesis of our study prompted us to consider the effects of verapamil, lithium or prednisone on expression genes involved in hypothalamic functioning as the parameter of reference. Unfortunately, we have been unable to find studies reporting this information. This precluded the possibility of defining an a priori hypothesis, as well as performing a sample size calculation. On this basis, to carry out our exploratory study, we chose a number of animals of six mice per group. Two independent experiments with six animals per group for each time point ( 8 am and $8 \mathrm{pm}$ ) were carried out. Animals were randomised (generating five groups by the RAND function of Excel software) and treated with verapamil, lithium, prednisone, amitriptyline or vehicle (control group) for 15 days. Upon a 15-day drug exposure, mice ( $\mathrm{n}=12$ per group) were sacrificed at 8:00 am and 8:00 pm by means of isoflurane anaesthesia, transcardial cold saline perfusion followed by immediate decapitation. At each time point a control group $(\mathrm{n}=12)$ was also sacrificed. Brains were then frozen at $-80^{\circ} \mathrm{C}$ upon the sacrifice and cut with a cryostat to collect hypothalamic regions. Specifically, bilateral anterior, lateral, and posterior hypothalamic regions were excised from frozen $20 \mu \mathrm{m}$ serial coronal sections according to the stereotaxic atlas of the mouse brain. Genes of interest were analysed in that hypothalamic region where they are physiologically more expressed.

\section{Quantitative PCR}

Total RNA was isolated from hypothalamic regions using Trizol Reagent (Life Technologies, Monza, Italy). One $\mu \mathrm{g}$ of RNA was retrotranscribed using iScript (Bio-Rad, Milan, Italy). RT-PCR was performed as reported (5). The primers used are reported in Figure 2.

\section{Statistical analysis}

Data are expressed as mean $\pm \mathrm{SEM}$. To test the difference between more than two groups ANOVA plus Tukey's post hoc test (according to KolmogorovSmirnov test for normality) was used. Differences were considered to be significant with a $p$-value $<0.05$. Statistical analyses were carried out using GraphPad Prism (version 7).

\section{Results}

\section{Effects of $\mathrm{CH}$ preventatives and amitriptyline on hypothalamic gene transcripts}

In order to adopt a clinically-relevant treatment protocol, mice were exposed to daily, oral drug administration for 15 days, a length of time sufficient for a $\mathrm{CH}$ preventative to prompt a therapeutic effect. Similarly, drugs were administered according to routinely adopted treatment schedules. Specifically, according to human-to-animal dose conversion guidelines and chronic treatment (6), verapamil and lithium carbonate were administered three times per day (8:00 am, $1: 00 \mathrm{pm}$ and 8:00 pm) at $3.4 \mathrm{and} 13 \mathrm{mg} / \mathrm{kg}$, respectively, whereas prednisone was dosed at $3 \mathrm{mg} / \mathrm{kg}$ at $8.00 \mathrm{am}$. An additional group of mice was exposed to oral amitriptyline $(1 \mathrm{mg} / \mathrm{kg}$ at 8:00 pm), a migraine preventative altering some hypothalamic functions here adopted as a negative control. Collectively, drug doses were about three-fold higher than those routinely adopted in patients, in keeping with standard conversion from human to mouse chronic dosing. Drug treatments were well tolerated, showing no effects on body weight and food/water consumption (not shown). 
As far as clock genes of the anterior hypothalamus are concerned, we found that none of the $\mathrm{CH}$ preventatives altered transcript levels of Clock, Bmall, Cryl and -2, as well as Perl and -2. Interestingly, mice receiving amitriptyline showed reduced Clock mRNA levels at both 8 am and $8 \mathrm{pm}$ and an increase of those of
Bmall at 8 am (Figure 1(b)-(g)). In the lateral hypothalamus, we evaluated transcripts for hypocretin/ orexin and melanin-concentrating hormone, two neurotransmitters with key roles in sleep regulation (7). We found that none of the tested drugs altered hypocretin transcripts, whereas only prednisone increased those of

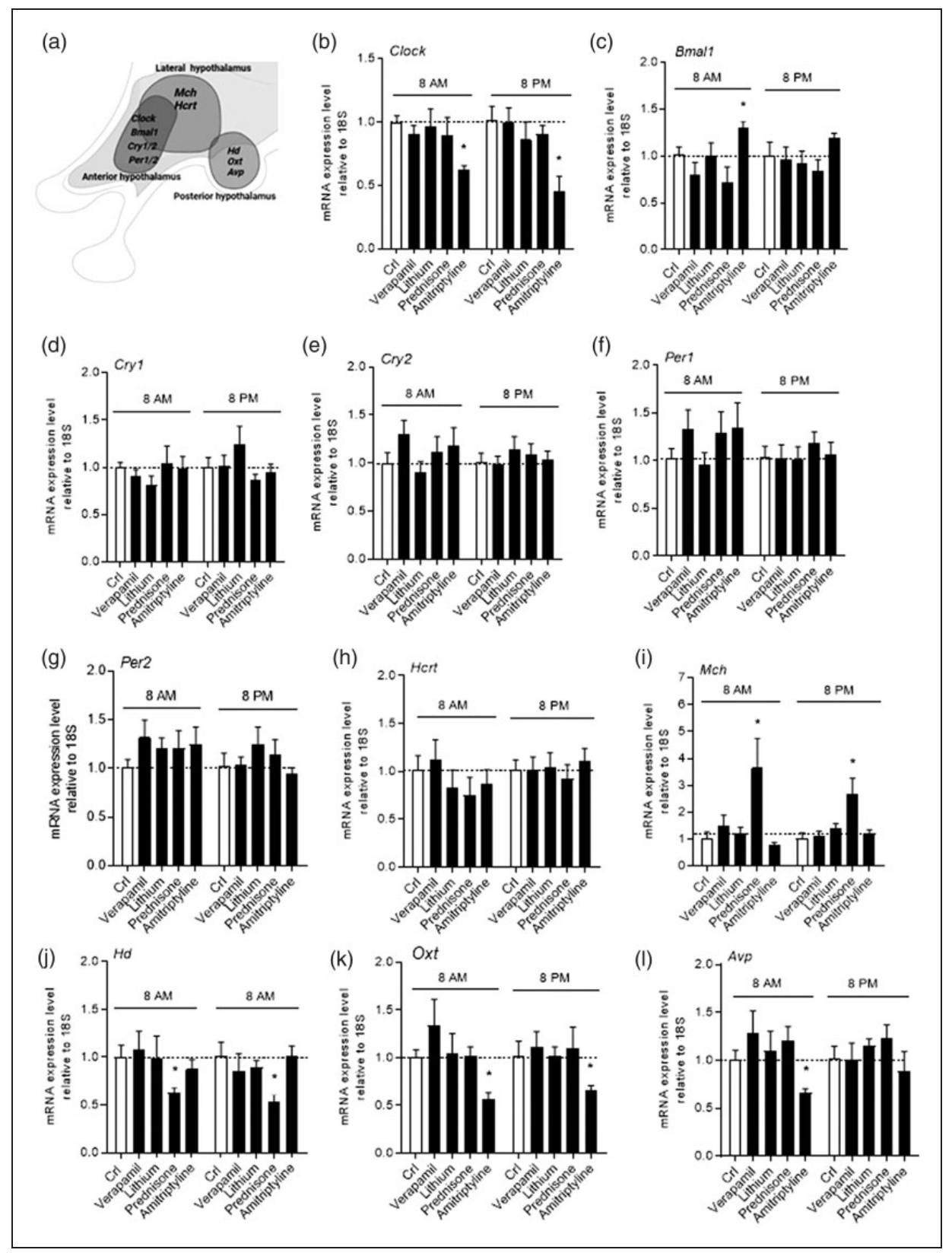

Figure I. Effects of verapamil, lithium, prednisone, and amitriptyline on gene transcripts involved in hypothalamic chronobiology. The hypothalamic regions (anterior, lateral and posterior) analysed and the respective genes is shown in (a). The impact of a chronic ( I 5 days), oral treatment with verapamil $(3.4 \mathrm{mg} / \mathrm{kg}$, three times per day), lithium carbonate ( $13 \mathrm{mg} / \mathrm{kg}$, three times per day), prednisone (3 mg/kg) or amitriptyline (I mg/kg) on transcript levels of Clock (b), Bmall (c), Cryl (d), Cry2 (e), Perl (f), Per2 (g), orexin Hcrt (h), melanin concentrating hormone Mch (i), histidine decarboxylase Hd (J), oxytocin Oxt (K) and arginine vasopressin Avp (L) within the mouse hypothalamus at 8 am and $8 \mathrm{pm}$ after 15 days of treatment is shown. Each column represents the mean \pm SEM of two experiments with six animals per group.

$*_{p}<0.05$ vs. Crl (ANOVA plus Tukey's post-hoc test). 


\begin{tabular}{|c|c|}
\hline Name & Sequence $5^{\prime}-3^{\prime}$ \\
\hline \multirow{2}{*}{ Clock } & FW-GACTGCTCCTGTAGCTTGTGG \\
\hline & RV-AAGCTGCTGTTCCTGGGAACT \\
\hline \multirow{2}{*}{ Bmal1 } & FW-GCCTITTGGGAAGGAACGAGATGCAG \\
\hline & RV-TAACCGGAAGTCGAGTCTTGG \\
\hline \multirow{2}{*}{ Cry1 } & FW-GCCTITTGGGAAGGAACGAGATGCAG \\
\hline & RV-GTGATGGTGTCTGCTGGCATCTCC \\
\hline \multirow{2}{*}{ Cry2 } & FW-CAAAGGACTACGGCTCCACGACAAC \\
\hline & RV-GGACTACAAACAGACGCGAATTCAGC \\
\hline \multirow{2}{*}{ Per1 } & FW-CTGATGCAAACAGCAATGGCTCAAGTG \\
\hline & RV-ACTCAGGAGGCTGTAGGCAATGGAG \\
\hline \multirow{2}{*}{ Per2 } & FW- CCAGCACGCTGGCAACCTTGAAG \\
\hline & RV-AGGGACACAGCCACAGCAAACATATC \\
\hline \multirow{2}{*}{ Hcrt } & FW-CCCCTGAGCTCCAGGCACCATGAAC \\
\hline & RV-ACCCGCAGCGTGGTTGCCAGT \\
\hline \multirow{2}{*}{ Mch } & FW-AAGATCCGTTGTCGCCCCTTCTCTGG \\
\hline & RV-GCATTCTGAACTCCATTCTCAGCTGGG \\
\hline \multirow{2}{*}{ Hd } & FW-TGCAACAGTGTGCCTATGAGTGCCC \\
\hline & RV-CTGGCGAGCACACTGAGAACTGC \\
\hline \multirow{2}{*}{ Oxt } & FW-GGCCTGCCCCAGTCTCGCTTGCT \\
\hline & RV-CCACGAAGCAGCCCAGCTCGTC \\
\hline \multirow{2}{*}{ Avp } & FW-CGCCAGGATGCTCAACACTACGC \\
\hline & RV-GCAGCAGATGCTTGGTCCGAAGC \\
\hline \multirow{2}{*}{$18 s$} & FW-AAAACCAACCCGGTGAGCTCCCTC \\
\hline & RV-CTCAGGCTCCCTCTCCGGAATCG \\
\hline
\end{tabular}

Figure 2. Primers used are shown in this table.

melanin-concentrating hormone at both 8 am and $8 \mathrm{pm}$ (Figure 1(h) and (i)). Within the posterior hypothalamus, we evaluated mRNA levels of histidine decarboxylase, the rate-limiting enzyme in the synthesis of brain histamine with a key role in CNS arousal/wakefulness (8), as well as the two neurohypophysis hormones oxytocin and arginine vasopressin. Figure 1(j) shows that histidine decarboxylase transcripts were reduced in mice receiving prednisone at both time points, but unchanged in animals challenged with the other preventatives. Conversely, amitriptyline reduced expression levels of oxytocin at 8 am and pm, whereas those of arginine vasopressin were reduced at 8 am only (Figure 1(k) and (1)). To rule out that lack of effects on gene transcripts being due to lack of power (see Methods), we conducted a post-hoc power analysis using the software G*Power 3.1.9.4, with ANOVA one-way test, and considering a beta/alpha ratio of 4 , the sample size ( 12 mice) and effect size reached for each single gene. We obtained a power of 0.78 for Clock, 0.84 for Bmal1, 0.75 for Cry1, 0.79 for Cry2, 0.73 for Per1, 0.82 for Per2, 0.49 for Hcrt, 0.96 for Mhc, 0.84 for $H d$, 0.81 for $O x t, 0.79$ for $A v p$. These findings indicate that power was reached for most of the gene.

\section{Discussion}

Even though pathophysiology of $\mathrm{CH}$ is largely unknown, both clinical evidence and functional neuroimaging point to a key role of the hypothalamus in disease pathogenesis. Given the preventative nature of verapamil, lithium, and prednisone in $\mathrm{CH}$ therapy, it makes sense that these drugs act within the CNS to reduce attack frequency. Indeed, all the three preventatives are known to cross the blood-brain barrier and affect CNS functioning. We reasoned that the three drugs may share the ability to alter hypothalamic homeostasis thereby altering the expression of genes involved in circadian rhythm and sleep/wake cycle. Interestingly, Clock and Cry nucleotide polymorphisms segregate with $\mathrm{CH}$ patients $(9,10)$. Similarly, genetic susceptibility loci are present in $\mathrm{CH}$ patients, even though they do not seem to be specifically related to hypothalamic function regulation (11). Together, these findings corroborate the relevance of specific chronotypes/gene association in $\mathrm{CH}$ pathogenesis. We found, however, that neither verapamil nor lithium affected transcript levels of mouse hypothalamic clock genes/neuropeptides, whereas prednisone 
increased melanin-concentrating hormone mRNAs and reduced those of histidine decarboxylase. Taken together, these findings indicate that the three preventatives do not alter the interconnected, transcriptiontranslation feedback loops underpinning clock gene expression within the mouse suprachiasmatic nucleus, the hypothalamic pacemaker of circadian oscillations. This is in keeping with the notion that verapamil exerts its prophylactic effect even when acutely administered (12). In this regard, however, it is worth noting that verapamil is rapidly extruded from the CNS by P-glycoprotein, an event that, in principle, might reduce its potential impact on hypothalamic gene expression. Conversely, corticosteroid therapy is well known to affect sleep, mostly causing sleep difficulties. We report here that, in keeping with its transcriptionregulating properties, prednisone increased melaninconcentrating hormone and reduced histidine decarboxylase transcripts within the lateral and posterior mouse hypothalamus, respectively. Oddly, these changes should promote rather than reduce sleep. Indeed, the melanin-concentrating hormone increases both REM and non-REM sleep, whereas diminished expression of histidine decarboxylase impairs histamine-dependent CNS arousal and wakefulness. We reason that these somehow paradoxical transcriptional changes occur as a rebound phenomenon in chronically exposed mice, a hypothesis in line with the transient nature of corticosteroid-dependent sleep disturbances. The inability of lithium to affect gene expression within the mouse hypothalamus was in part unexpected. This is not only because of the efficacy of lithium in bipolar disorder, a psychiatric condition typically characterised by specific chronoperiods, but also in light of the drug's impact on rodent behavioural rhythms (13). To our knowledge, however, no studies have investigated the effect of the drug on clock gene expression in wild-type mice. Of note, our findings are at odds with a recent study by Burish and colleagues (14) reporting slight changes of circadian clock gene transcripts in the hypothalamus of mice exposed to verapamil. Possibly, this apparent inconsistency can be explained considering that the authors adopted a high dose of verapamil ( $\sim 6.5$-fold higher than that clinically relevant), a limited number of mice per group, as well as drug administration in drinking water rather than reproducing a clinically relevant treatment schedule. Surprisingly, amitriptyline, adopted here as a putative negative control, altered hypothalamic transcript levels of Clock, oxytocin, and arginine vasopressin. When put into a clinical perspective, the ability of amitriptyline to alter hypothalamic transcriptional homeostasis may underpin its antimigraine properties, as well as its modest benefit in $\mathrm{CH}$ treatment (15). The ability of amitriptyline to reduce oxytocin and arginine vasopressin expression can be related to the drug's impact on peripheral aminergic neurotransmission. Of note, in keeping with our findings, severe hypotension during amitriptyline intoxication only responds to arginine vasopressin (16).

In conclusion, our data suggest that $\mathrm{CH}$ preventatives act upstream or downstream from the hypothalamus, possibly targeting signalling pathways independently involved in disease pathogenesis. However, given that the present findings only provide evidence for lack of diurnal variation in hypothalamic gene expression, further studies are needed to rule out that $\mathrm{CH}$ preventatives prompt circadian alterations showing a different timescale.

\section{Article highlights}

- It is currently unclear whether cluster headache $(\mathrm{CH})$ preventatives modify hypothalamic transcriptional homeostasis.

- We report that prolonged exposure to verapamil, lithium or prednisone does not affect expression of mouse hypothalamic genes regulating chronobiology.

- $\mathrm{CH}$ preventatives may act upstream or downstream from the hypothalamus.

\section{Declaration of conflicting interests}

The authors declared no potential conflicts of interest with respect to the research, authorship, and/or publication of this article.

\section{Funding}

The authors disclosed receipt of the following financial support for the research, authorship, and/or publication of this article: This work was supported by grants from Health
Project 2018 Region of Tuscany, and PRIN2017 Italian Ministry of the University and Research (2017JL8SRX8).

\section{References}

1. Wei DY and Goadsby PJ. Cluster headache pathophysiology - insights from current and emerging treatments. Nat Rev Neurol 2021; 17: 308-324.

2. May A, Schwedt TJ, Magis D, et al. Cluster headache. Nat Rev Dis Primers 2018; 4: 1-17. 
3. May A. The role of imaging in the pathophysiology and diagnosis of headache. Curr Opin Neurol 2005; 18: 293-297.

4. Jürgens TP, Leone $\mathrm{M}$, Proietti-Cecchini A, et al. Hypothalamic deep-brain stimulation modulates thermal sensitivity and pain thresholds in cluster headache. Pain 2009; 146: 84-90.

5. Buonvicino D, Urru M, Muzzi M, et al. Trigeminal ganglion transcriptome analysis in 2 rat models of medication-overuse headache reveals coherent and widespread induction of pronociceptive gene expression patterns. Pain 2018; 159: 1980-1988.

6. Nair AB and Jacob S. A simple practice guide for dose conversion between animals and human. J Basic Clin Pharm 2016; 7: 27-31.

7. Concetti $\mathrm{C}$ and Burdakov D. Orexin/hypocretin and $\mathrm{MCH}$ neurons: Cognitive and motor roles beyond arousal. Front Neurosci 2021; 15: 294.

8. Yoshikawa T, Nakamura T and Yanai K. Histaminergic neurons in the tuberomammillary nucleus as a control centre for wakefulness. Br J Pharmacol 2021; 178: 750-769.

9. Fourier C, Ran C, Zinnegger M, et al. A genetic CLOCK variant associated with cluster headache causing increased mRNA levels. Cephalalgia 2018; 38: 496-502.
10. Fourier C, Ran C, Sjöstrand C, et al. The molecular clock gene cryptochrome 1 (CRY1) and its role in cluster headache. Cephalalgia 2021; 41: 1374-1381.

11. O'Connor E, Fourier C, Ran C, et al. Genome-wide association study identifies risk loci for cluster headache. Ann Neurol 2021; 90: 193-202.

12. Petersen AS, Barloese MCJ, Snoer A, et al. Verapamil and cluster headache: Still a mystery. A narrative review of efficacy, mechanisms and perspectives. Headache 2019; 59: 1198-1211.

13. Moreira $\mathbf{J}$ and Geoffroy PA. Lithium and bipolar disorder: Impacts from molecular to behavioural circadian rhythms. Chronobiol Int 2016; 33: 351-373.

14. Burish MJ, Han C, Mawatari K, et al. The first-line cluster headache medication verapamil alters the circadian period and elicits sex-specific sleep changes in mice. Chronobiol Int 2021; 38: 839-850.

15. Visvanathan $\mathrm{N}$, Chinnu $\mathrm{M}$ and Mahadevan $\mathrm{R}$. Amitriptyline in the management of cluster headache (P3.055). Neurology 2015; 84. ISSN: 1526-632X.

16. Barry JD, Durkovich DW and Williams SR. Vasopressin treatment for cyclic antidepressant overdose. J Emerg Med 2006; 31: 65-68. 